\title{
La investigación en la formacion "Web-Learning"
}

Guillermo Vázquez, David Riley, Ramón L. Cuervo

Fundacion IAVANTE, Consejería de Salud de Andalucía

La educación médica tiene en el Web Learning, una herramienta que permite el acceso universal a la formación y al entreno. El Web Learning, supone también, un reenfoque y redimensionado de la educación en todas sus dimensiones incluyendo la tecnológica. Todo lo anterior junto con las cuantiosas inversiones que supone la implantación del Web Learning, aconseja que la investigación para identificar el proceso óptimo de implementación y desarrollo, se convierta en uno de sus pilares de referencia. En este artículo se explicitan una serie de preguntas relativas a los diversos aspectos del Web Learning que a criterio de los autores deben de ser contestados a través de la investigación, así como aquellas metodologías científicas mas apropiadas a las preguntas formuladas. La revisión tanto bibliografía, como los libros de ponencias y comunicaciones de congresos nacionales, evidencia la ausencia casi total de investigación en este campo de la medicina, lo cual se agrava con la carencia de becas para financiar estos estudios. Solo una estrategia global puede superar esta situación y promover la educación basada en la mejor evidencia.

Palabras Claves: Educación, Online, e.Learning, TICs, Telemedicina

Correspondencia:

G. Vázquez

Director de I+D+i Fundacion IAVANTE

Parque Tecnológico de Ciencias de la Salud

Avda de la Ciencia s/n

18.100 Armilla; Granada

Email: guillermo.vazquez@iavante.es

D. Riley: david.riley@iavante.es

R. L. Cuervo: ramon.lcuervo@iavante.es

\section{UNA PERSPECTIVA GENERAL DE LA EDUCACIÓN MEDIANTE WEB-LEARNING}

El Web-learning, conocido con otros nombres tales como Online Learning o también e.Learning, y distance learning, representa una modalidad de enseñanza y aprendizaje a distancia basada en las Tecnologías de la Información y Comunicación (TICs $)^{1}$. Esta forma de enseñanza a distancia se inicio en el siglo pasado utilizando inicialmente el correo, y sumándose posteriormente en los años 70, el empleo de la radio, siendo en nuestro país el mejor ejemplo la Universidad Nacional de Educación a Distancia. Esta Universidad no presencial, fue una acción innovadora en su tiempo, cuya principal característica fue promocionar la accesibilidad a la formacion superior a un gran número de personas que les era imposible acudir a las universidades clásicas. Actualmente Internet y en general las TICs, permiten acceder a la educación sin limites geográficos ni de tiempo, ajustándose bien a las necesidades individuales de aprendizaje ${ }^{1}$.

Técnicamente, las ventajas del Web-learning ${ }^{2,3}$ sobre el material escrito en papel se podrían resumir diciendo que puede combinar diferentes modalidades de estímulos sensitivos, como vista y oído, diversas combinaciones de animación en los contenidos, y finalmente una gran interactividad entre discente 
con el material docente, con los compañeros de curso y con los tutores a través de chats, forums, videoconferencia en tiempo real o diferidos, o bien con espacios colaborativos, tales como los Wikis Blogs o Podcast ${ }^{4}$. Finalmente el profesional / estudiante puede recibir y descargar la información en su ordenador personal, en una PDA, o en un iPod, y complementarlo con sus teléfonos móviles de tercera generación, y pronto en la propia pantalla de televisión domiciliaria. Este conjunto de posibilidades tecnológicas, no son mas que el soporte para conseguir incrementar la accesibilidad de las acciones educativas y de entreno.

\section{LOS FUNDAMENTOS ACTUALES DE LA EDUCACIÓN}

La Educación y sus diferentes vertientes se han desarrollado hasta las últimas décadas basandose en los paradigmas de arte, clase magistral, memorización, formacion centrada en los profesores, y conocimiento codificado. Los conceptos relacionados con la formacion basada en competencias, y centrada en el alumno junto con la necesidad de evaluar y medir, solo se han incorporado recientemente a la educación ${ }^{5}$. La educación basada en competencias, es decir el conjunto de conocimientos, habilidades y actitudes que permiten a un profesional realizar adecuadamente sus funciones, tiene como referente la Pirámide Educativa de Miller ${ }^{5}$. En dicha Pirámide se identifican cuatro escalones, los dos primeros relacionados con el conocimiento, el tercero con la práctica clínica y el cuarto con los resultados asistenciales. Paralelamente Kirkpatrick ${ }^{6}$ propone 5 niveles evaluativos de las acciones formativas; el primero se corresponde con los aspectos formales del curso, tales como la asistencia, el segundo con la satisfacción de los estudiantes / profesionales en relación a su percepción del curso, el tercero al impacto de la acción formativa sobre la práctica clínica y el quinto a la mejora de los resultados clínicos. La educación médica, al igual que otras ramas de la medicina debe de basarse en la evidencia mejor posible, y queda refrendada en la recién creada Colaboración Internacional de Educación Médica Basada en la Mejor Evidencia ${ }^{6}$.

\section{LOS CAMPOS DEL WEB-LEARNING ABIERTOS A LA INVESTIGACIÓN}

El concepto de la educación médica basada en la evidencia nos lleva de la mano al campo de la investigación en la educación médica ${ }^{7}, \mathrm{y}$ en nuestro caso a la investigación del Web Learning como instrumento formativo $^{8,9}$. La educación basada en un entorno Web, tiene una serie de componentes, cada uno de los cuales tiene su propio método de investigación y evaluación, y un diseño apropiado para su estudio ${ }^{10}$. De manera breve podemos encontrar en la tabla 1 sus principales componentes y preguntas de investigación. A su vez cada componente tiene peculiaridades específicas ${ }^{11}$, que intentaremos ver seguidamente. El primer componente son los software sobre los que va descansar la acción educativa ${ }^{9}$; tienen que reunir una serie de requisitos, entre los que destacan su accesibilidad, navegabilidad e interactividad, es decir su manejo fácil e intuitivo, junto una estructura y unos componentes que vayan mas allá de una mera copia del libro escrito. Este tipo de software educativo tiene diversas herramientas para su evaluación. La siguiente barrera a investigar es el nivel de digitalización que poseen los posibles usuarios de la plataforma de Web.learning, así como los idiomas a manejar. Una de las causas mas frecuentes de fracaso es, además de la dificultad de la propia Web, la falta de conocimientos de sus potenciales usuarios, así como la no accesibilidad a la tecnología necesaria ${ }^{1}$. Adecuar el software a dichos conocimientos y potenciar la formacion previa y el apoyo a lo largo de la acción formativa constituye otra variante a analizar, evaluar y cuidar. Los guiones y ejercicios sobre los que se construye la acción formativa deben de diseñarse según unos objetivos competenciales predeterminados $\mathrm{y}$ comprobando adecuadamente la validez y fiabilidad de la acción formativa ${ }^{3}$. Estos aspectos no se recogen bien en la literatura. El impacto de la acción formativa de e.Learning, utilizando la clasificación de Kirkpatrick como referencia, se obtiene de los estudios sobre la satisfacción de los participantes, pudiéndose encontrar numerosos cuestionarios que cubren los diversos aspectos de este tipo evaluación subjeti$\mathrm{va}^{8,11}$. A medida que avanzamos desde los estudios de satisfacción y educativos, hacia los estudios de objetivos de práctica clínica y resultados clínicos ${ }^{3,8}, \mathrm{su}$ número decrece rápidamente, siendo realmente escasos los que tratan de este último eslabón del impacto educativo. Esta situación se repite no solo con la edu- 
Tabla 1: Investigación / Evaluación en Web-learning aplicada a la Educación Médica

1. Eslabones

a. Software: Herramientas de diseño, y colaborativas. Navegabilidad y ergonomía. Software libre versus el propietario, ventajas e inconvenientes

b. Usuarios: Nivel de digitalización, barreras tecnológicas y culturales

c. Documentación y guiones de las acciones formativas:

- Combinación adecuada de la información auditiva y visual

- Adecuación de las herramientas de interactividad

- Contextualización y feedback

- Ubicación en la Pirámide de Miller

d. Objetivos cuantitativos:

- Número de alumnos, abandonos, visitas,

- Participación en actividades interactivas.

e. Objetivos de Satisfacción: Percepción subjetiva de curso, percepción de la calidad de los materiales y de los tutores.

f. Objetivos Educativos: asimilación conocimientos y habilidades y actitudes al final de curso.

g. Objetivos de Práctica Clínica: incorporación de los conocimientos, habilidades y actitudes adquiridos, a la práctica clínica.

h. Objetivos Clínicos: repercusión en los objetivos asistenciales.

2.-Principales áreas de investigación

a. Evaluación comparativa entre diversos modelos de software y su impacto en la formacion.

b. Efectividad / eficacia de diferentes modelos de retroalimentación

c. Validez y reproducibilidad de de guiones y cuestionarios docentes

d. Mejoras de las curvas de entrenamiento comparando diversos métodos educativos

e. Mejora de la práctica clínica / resultados clínicos, comparando resultados antes y después de una acción formativa

f. Mejora de la práctica clínica / resultados clínicos, comparando resultados obtenidos con métodos distintos en grupos distintos.

g. Investigación sobre modelos educativos y pedagógicos que modifiquen la práctica clínica

h. Investigación sobre resultados clínicos que puedan mejorarse eficientemente con las acciones educativas.

i. Relación entre calidad asistencial y educación y entrenamiento

cación online, sino también con todas las variantes de educación presencial. En los estudios de investigación que responden a las preguntas que figuran en la tabla 1, se pueden encontrar algunos con diseños observacionales, pero son realmente escasos los que tienen formato experimental ${ }^{12,13,14}$. En la tabla 2, se pone en evidencia esta situación. Durante el periodo 2000-2005, utilizando Medline como fuente básica de información, los trabajos relacionados con la educación médica se aproximan de los cincuenta mil, pero sin embargo solo el doce por ciento se dedican a la formacion continuada y el uno y medio por ciento a la formacion online. Cuando la formación online se analiza desde el punto de vista del número de estudios con diseños randomizados o de estudios relacionados con resultados clínicos, se localizan menos del uno por ciento, y que combinen ambos aspectos solo encontramos 2 trabajos. Estas cifras reflejan que la investigación en educación médica en sus diversos eslabones y las metodologías adecuadas para hacerlo están en un estado incipiente, y posiblemente no es percibida por el público como una necesidad científica. Esta situación permite creer que es necesaria una estrategia que las implemente lo más rápidamente posible. En la sociedad del conocimiento del siglo XXI, la adquisición de dicho conocimiento se convierte en una prioridad de máximo rango, y la investigación para identificar las mejores prácticas docente sobre las que aplicar la educación basada en la evidencia se convierte en una necesidad ineludible.

Tabla 2: Diferentes Modalidades de Educación y su relación con la investigación

\begin{tabular}{ll}
\hline Palabra clave & número de trabajos (\%) \\
\hline Ensayos randomizados (ER) & 93476 \\
Resultados clínicos (RC) & 90687 \\
Educación médica (EM) & $44100(100 \%)$ \\
Formacion Continuada (FC) & $5203(12 \% *)$ \\
Formacion Online (FO) & $\left.538(1.5 \%)^{*}\right)$ \\
Educación Medica + ER & $1397\left(3.16 \%{ }^{*}\right)$ \\
Formacion Continuada +ER & $178\left(0.4 \% 0^{*}\right)$ \\
Formacion Online + ER & $21\left(0.04 \%{ }^{*}\right)$ \\
Educación Médica + RC & $1653(3.7 \% *)$ \\
Formacion Continuada + RC & $212\left(0.05^{*}\right)$ \\
Formacion Online + RC & $22\left(<0.005^{*}\right)$ \\
Formacion online + ER + RC & $2\left(<0.005^{*}\right)$
\end{tabular}




\section{LOS OBJETIVOS DE INVESTIGACIÓN CON LA FORMACIÓN WEB-LEARNING}

La formacion online, ha demostrado que puede ser utilizada no solo para conocimientos a memorizar e interpretar, sino que sirve para plantear situaciones donde se deba de utilizar y desarrollar el pensamiento crítico y reflexivo ${ }^{3,4}$. También ha demostrado su utilidad para adquirir habilidades en las que el componente visual sea predominante tal es el caso de las imágenes radiológicas de toda índole, dermatológicas o microscópicas ${ }^{3,11}$. Aunque existen pocos trabajos que versen sobre el impacto de la formacion online sobre la práctica clínica, en el año 2005, M Fordis ${ }^{14}$ publicó un trabajo que se puede considerar un hito en el campo de la investigación de formacion en Internet y su impacto en la práctica clínica. En primer lugar demostró que es posible aplicar una metodología experimental depurada en el campo de la educación online, y en segundo lugar, objetivo que este tipo de formacion es igual y en ocasiones superior a la formacion continua, en relación a su impacto en la práctica clínica y que transcurrido un año dicha mejora persiste. Los resultados a investigar en relación con la práctica clínica ${ }^{11}$ pueden resumirse en los siguientes puntos: volumen de pacientes atendidos, prescripción farmacológica adecuada, cuidados aplicados adecuados, mejora en los tiempos asistenciales, mejora en comunicación paciente /médicos /familia, o mejora en costos y utilización de recursos. En relación con los resultados asistenciales, los puntos en los que puede impactar son la mortalidad, la calidad de vida, disminución de efectos adversos, y la seguridad del paciente. La evaluación de estos resultados, se puede hacer online especialmente para el campo del conocimiento en sus diversas facetas, y para algunas habilidades visuales citadas. Sin embargo la evaluación de la práctica clínica y de los resultados clínicos requiere otros instrumentos tales como portafolio, exámenes clínicos objetivos estructurados, o evaluación por pares. El diseño de la investigación educativa, puede requerir diferentes tipos de diseño, dependiendo de la pregunta responder: en algunas ocasiones pueden servir estudios observacionales ${ }^{11}$ en sus distintas modalidades, y en otras puede ser imprescindible aplicar diseños experimentales; algunos estudios, especialmente cuando se dirigen a campos en los que el componente subjetivos es importante, pueden requerir estudios cuantitativos, difiriendo en este caso de los estudios de investigación mas frecuentes en los campos clínicos.

\section{LAS BARRERAS QUE DIFICULTAN LA INVESTIGACIÓN EN EDUCACIÓN MÉDICA}

Las causas por las cuales la educación en medicina, y en mayor medida aún la educación online, no se considera un área de investigación similar a otras áreas clásicas de investigación en medicina pueden ser muy variadas. En primer lugar, los profesionales no visualizan la educación mas allá de una herramienta bien establecida para transferir el conocimiento; esta visión se basa generalmente en una visión centrada en el profesor y en la memorización de contenidos. El desconocimiento por muchos profesionales de las tendencias pedagógicas actuales, de las nuevas metodologías de educación y entreno basadas en las competencias, y el de los campus virtuales multimedia con sus posibilidades de espacios colaborativos, pudiera ser una de los factores claves para entender la ausencia de la educación como área de investigación. En la tabla 3 queda patente que la educación, las TICs, y espacios próximos como es la telemedicina, no se incorporan en las comunicaciones de las Sociedades Científicas Médicas, pero tampoco en las mesas redondas de las mismas sociedades.

Tabla 3: Congresos de Sociedades Científicas durante el año 2005

\begin{tabular}{lll}
\hline Sociedad Científica & Comunicaciones & TICs/Educación \\
SEMICYUC* & 425 & $9(2.1 \%)$ \\
SEMES** & 756 & $35(4.6 \%)$ \\
SEMI $^{* * *}$ & 1575 & $14(8 \%)$ \\
SECA $^{* * * *}$ & 821 & $27(3.2 \%)$
\end{tabular}

\footnotetext{
*Sociedad Española de Medicina Intensiva, Crítica y Unidades Coronarias **Sociedad Española de Medicina de Urgencias y Emergencias ***Sociedad Española de Medicina Interna

****Sociedad Española de Calidad Asistencial
}

Pie de Tabla: Algunos conceptos analizados globalmente con las palabras claves adecuadas, ponen en evidencia la poca penetración de la educación como un campo especifico de trabajo de las sociedades científicas 
Esto a su vez es un reflejo de la escasez de trabajos de investigación. Uno de los grandes promotores de la Calidad Asistencial, Avedis Donabedian ${ }^{15}$, incorpora en el año 2005, la educación médica como uno de los eslabones esenciales del ciclo de la calidad asistencial, mientras que la tendencia actual de incrementar la seguridad de los pacientes, también considera la educación médica como una de sus herramientas imprescindibles. Por tanto, es fácil deducir, que existen suficientes estímulos externos para que este déficit en investigación educativa de las sociedades científicas se vaya corrigiendo próximamente.

Un segundo aspecto es la falta de financiación, las convocatorias de ayudas a la investigación en Sanidad no suelen contemplar la educación, y lo acercan a la telemedicina. Las convocatorias clásicas del Fondo del Investigación Sanitaria del Instituto Carlos III de los últimos años, no tiene ningún capítulo específico para la investigación en los diversos campos de la educación médica. Actualmente la única manera posible es a través de la relación indirecta entre TICs telemedicina y e.Learning. Esto contrasta las diversas posibilidades que la educación en general y por tanto la médica también a través del VI Programa Marco de la UE en el comisariado de Educación y Cultura, especialmente en alguno de los subapartados del Programa Sócrates como es el subprograma Minerva, y que tendrá continuidad en el VII Programa Marco en el programa denominado "Lifelong Learning"; estas herramientas tienen como base la innovación pedagógica, y esta innovación conlleva el cambio.

Todo lo expuesto permite visualizar la formacion basada en "Web Learning", como un instrumento en plena expansión, llamado a sustituir a la formacion presencial en muchos campos y áreas, y que para su aceptación definitiva en el campo de la educación médica, deberá de basarse en estudios que permitan objetivar la mejor manera de aplicarse. En este trabajo se pone de manifiesto la necesidad de una estrategia clara y bien definida para fomentar la investigación en educación médica en todas sus facetas, pero muy especialmente en su vertiente Web Learning.

\section{BIBLIOGRAFIA}

1. J Mckimm, C Jollie, P Cantillon. ABC of learning and teaching Web Based Learning. BMJ 2003; 326:870-873

2. T. Greenhalgh. Computer assisted learning in undergraduate medical education. BMJ 2001; 322:40-44

3. J Ruiz, Mj Mintzer, Rc Leipzig. The Impact of e.Learning in medical education. Acd. Med 2006; 86:207-212

4. MN Boulos, I Maramba, S Wheeler. Wiki, blogs, and podcasts, a new generation of web based tools for individual collaborative practice and education. BMC Medical Education 2006; 6:41-49

5. J Norcini. ABC of learning and teaching in medicine work based assessment. BMJ 2003; 326:753-755

6. A Centeno, JM Martinez-8Carretero. Innovaciones, investigación y evidencias en educación médica. La Colaboración EMBE (Educación Médica Basada en la mejor Evidencia). Educación Med. 2003; 6:26-30

7. Bligh J, Parsell G. Research in medical education: finding its place. Med Educ 1999;33:162-3.

8. Education Group for Guidelines. on Evaluation. Guidelines for evaluating papers on educational interventions. BMJ 1999; 318: 1265-1267

9. T. Grunwald, Ch Corsbie-Massay. Guidelines for cognitive Efficient Multimedia Learning Tolls: Educational Strategies, Cognitive Load, and Interface Desgin. Acade Med 2006; 81:213-223

10. G Regehr * The AAMC: GEA Research in Medical Education (RIME) Conference 2003. Medical Education. 38(7):791-792, July 2004.

11. D. Reed, EG Price, D Windish, S M Wrigt, A Gozu, E. Hsu and als Challenges in Systematic Reviews of Educational Intervention Studies. Ann Intern Med 2005, 142:1080-1089

12. B Haynes, Can it work? Does it work? Is it worth it?. The testing of healthcare interventions is evolving. BMJ 1999; 19: 652-653

13. JL Uribe, W Ralph, AY Glaser, M Fried. Learning curves, acquisition and retention of skills trained with the endoscopic surgery simulator. Am J Rhinol 2004; 18:87-92

14. M Fordis, J King, Ch Ballantyne, P Jones, K Shneider, S B Spann and als. Comparison of the instructional efficacy of internet-based CME with live interactive CME workshops. A randomized controlled trial. JAMA 2005: 294: 1043-1051

15. A Donabedian. An introduction to Qualtiy Assurance in Health Care. Edited by Oxford University Press 2003, New York 